\title{
Rician Noise Removal and Straightening of Blood Vessel in MR Images
}

\author{
${ }^{1}$ Jayabal Papitha and ${ }^{2}$ Damodaran Nedumaran \\ Central Instrumentation and Service Laboratory, University of Madras, Guindy Campus, \\ Guindy, Chennai, Tamilnadu, INDIA \\ ${ }^{1}$ dnmaran@gmail.com, ${ }^{2}$ dnmaran@yahoo.com
}

\begin{abstract}
Purpose: To improve the visualization of severities in blood vessels combined Smoothing and straightening of a blood vessel was attempted for effective diagnosis of tumors and platelet formation in blood vessels.
\end{abstract}

Method: Fast gradient projection method was used for removing the Rician noise present in MR images. The smoothed image was extracted using the binarization technique and the extracted blood vessels are straightened using a tangent function.

Result: These techniques were tested in a variety of MR images and the results of this study reveal that the proposed method removed the Rician noise without affecting the diagnostic details and compared with existing methods quantitatively, extracted and straightened the blood vessels for making a clear decision about the severity of the disease.

Conclusion: The smoothed, extracted and straightened images are very much useful for the diagnosis of tissue characterization and tumor detection.

Keywords: Rician noise, Fast gradient projection, Extracting, Centerline, Straightening

\section{Introduction}

Magnetic resonance imaging (MRI) is an outstanding imaging modality but suffers from low contrast and noise during image acquisition and transmission. The noise in magnitude MR images is accurately modeled by a Rician distribution and becomes tough to diagnose accurately for Image analysis and tissue charactrization.

Vessel extraction is employed as a potential tool for automatic clinical diagnosis, treatment planning, computer-integrated surgery, quantification of tissue volume, localization of pathology, and registration using imaging modalities like Magnetic Resonance Imaging (MRI), Computed Tomography (CT), X-ray Angiography (XRA), Magnetic Resonance Angiography (MRA), etc. With the developments in computer technology and imaging technology, the automation of imaging diagnostic has been implemented to handle the volume of data involved in the imaging modalities and to reduce the cost of diagnosis. Blood vessel extraction is the most important automation technique due to its effective and frequent 
Jayabal Papitha and Damodaran Nedumaran; Rician Noise Removal and Straightening of Blood Vessel in MR Images. Journal of Biomedical Engineering and Medical Imaging, Volume 2, No 6, December (2015), pp 28-38

applications, viz., vascular diseases diagnosis, surgery planning, and post-therapeutic monitoring. Various approaches are reported in the in the literature for the extraction of the vessel structures from MR images, which have their own limitations. Some images cannot provide an entire vessel in one slice. In that case, a smooth curved surface can be used to fit into the winding vessel and cut open the volume along the centerline of a vessel instead of using a straight cut plane. This allows bends in a vessel to be straightened, so that the entire length can be visualized in one image. The vessel interior is of great importance for evaluating the degree and distribution of diseases. Visualization techniques re-sample a longitudinal cross-section along the medial axis in one image plane for simultaneous and accurate visualization of diagnostic features. Different strategies have been integrated to achieve different visualization techniques. Most advanced medical workstations are able to semi-automatically find a centerline between two points specified by the user. On the visualization side, some of the aspects of interest are: Variation of seeding structures, Integration length, Type of primitive for an integrationbased visualization, Different time steps of the flow, Variation of other visualization parameters. Several vessel visualization techniques have been used in CT images, whereas for magnetic resonance (MR) images the application of these techniques is limited due to the presence of inherent Rician noise. As a result, we implemented the fast gradient projection algorithm for the removal of Rician noise present in MR images and extracted and straightened the blood vessel using binarization and visualization techniques, respectively. MATLAB is used for algorithm development due to its versatility in implementing the complex operations very easily using the built-in signal and image processing toolkits.

The rest of this paper is organized as follows: In Section 2, we discuss the functionality and applications of vessel straightening in various imaging modalities. Section 3 presents the basics of Fast Gradient Projection Method and related algorithms. Section 4 briefly reviews the centerline computation and straightening techniques. The results and the concluding remarks of this study are given in Sections 5 and 6 , respectively.

\subsection{Related Work}

O'Donnel et al. [1] proposed a piecewise polynomial functions connected at knot points for a simple spine model based on cubic B-splines. The tangent of the spine at each knot point was calculated and aligned with the tangent of the succeeding knot point with a minimal rotation in order to construct a discrete frame at each knot point. Then the frames were joined together by interpolation based on the EGC model. Barrett and Carvalho developed a software tool for straightening curved chromosomes [2]. Zhang et al. generated a curved multiplanar reformation (MPR) images for vessel segmentation and tracking for the gradation of the calcified coronary artery plaque [3]. Chuang and Chen developed a straightening tool for a coronary artery that provides slices of the straightened artery at any crosssectional angle, and more information about the coronary artery and the surrounding tissues [4]. Kanitsar et al. proposed a helical curved planar reformation (CPR) technique to visualize the interior of a vessel and an untangled CPR technique for displaying the unobscured display of a vascular tree that was independent of the viewing direction $[5,6]$. Wang analyzed the extraction of the 3D centreline of the vessel of interest through a vessel tracking algorithm and created four longitude vascular volumes at 45응 intervals for the visualization of the curved arteries using a multiplanar reformation (MPR) method [7, 8]. Zhang addressed the coronary vessel tracking problem by extracting the vessel centerlines as intensity ridges from CTA images using a ridge traversal algorithm [9]. Wink et al. presented several techniques for the extraction and display of coronary center line for the development of automatic 
diagnostic cath for the early and accurate detection of coronary artery disease [10]. Saran et al. developed a novel method to remove the Rician noise and RF inhomogeneity in MR images during segmentation using the Goldfeld, Quant, and Trotter (GQT) subtraction method [11]. Lawler demonstrated a combined tool to find the center of the vessel (centerline tools) and vesselstraightening of tortuous curves in coronary arteries [12]. Kanitsar proposed four different segmentation techniques for vessel straightening, viz., projected CPR, stretched CPR, straightened CPR, and helical CPR and three different methods for vessel tree visualization such as multi-path projected CPR, multipath stretched CPR, and untangled CPR with their pros and cons [13]. Kim developed 3-D volume extraction algorithm for segmentation of cerebrovascular structures on brain MRA that provided better results than other traditional methods [14]. Olabarriga et al. developed a Hessian-based filter to analyze the second-order variation in CT image intensity for determining the axis and type of local structure [15]. Zenteno et al. applied a distal-to-proximal technique for the segmentation of tortuous cervical segment in internal carotid artery for the management of complex interacranial aneurysms [16]. Dupej studied the shortfalls of the Straightened Curved Planar Reformation (SCPR) method and improved it by choosing equidistant samples on the vessel center line for calculating the local coordinate system and the square-shaped area (slices) of the volume centered at the center line was calculated, instead of short line segments in the case of SCPR. These slices were stacked to obtain a block of volume data using the maximum intensity projection (MIP) that provides more spatial context for more intuitive orientation in the resulting image [17]. Ropinski integrated a specialized multipath curved planar reformation and multimodal vessel flattening technique for the visualization of multiple data sets for achieving the undistorted diameter of the vessel as well as the positions of the arteries [18]. RivestHenault et al. developed a 2D/3D nonrigid registration algorithm based on the global affine transformation and local nonrigid registration methods for the automatic extraction of vessel centerlines of the coronary arteries and aligning with the bi-plane fluoroscopic angiograms [19]. Koocsis et al. developed an algorithm for filamentous particles straightening using a non-uniform cubic spline [20]. Wassermann et al. developed a Diffusion Tensor method to recover the bundles of fibers in the spinal cord and applied the Gaussian framework to recover the most representative fiber, which was used to interpolate and straighten the spinal cord fibers [21]. Angelelli and Hauser demonstrated the straightening and the visualization of tubular flow by aligning the center line of the bounding tubular structure with one axis of the screen defined as vector fields on a Cartesian grid [22]. Mistelbauer et al. proposed Curvicircular Feature Aggregation (CFA), which aggregates the rotated images into a single view by eliminating the rotation around the centerline of the vessel as in the case of the CPR method in order to view the vessels in one image [23]. Lee and Rasch developed an algorithm based on topological and orientation invariant visualization techniques for the visualization of vascular trees [24].

\section{Methods}

\subsection{Fast Gradient Projection Method}

Bound constrained optimization problems are solved using Gradient Projection (GP) methods which allow large changes in the data set in each iteration. Linear GP methods are applied in non-linear constrained programming functions. Goldstein [25] and Lewtin \& Polyak [26] introduced a GP method for resolving convexly constrained minimization problems as an extension of the steepest descent or a Cauchy algorithm for solving unconstrained optimization problems. The resulting curve obtained from 
Jayabal Papitha and Damodaran Nedumaran; Rician Noise Removal and Straightening of Blood Vessel in MR Images. Journal of Biomedical Engineering and Medical Imaging, Volume 2, No 6, December (2015), pp 28-38

the boundary region projected the negative gradient that can be used in conjunction with other methods with faster rates of convergence that reduces the steepest-descent on the subspace of free variables [27]. The GP method identifies the optimal active set in a finite number of iterations for bound constrained problem proposed by Bertsekas [28]. Later in 1982, Bertsekas derived some results for a projected Newton Method [29] and Gafini \& Bertsekas for a 2-metric projection method [30]. Dunn proved that the GP identifies the optimal active constraints in a finite number of iterations in a strict complementary condition [31]. The above statement was extended by Burke and More, and proved that under Dunn's non-degeneracy assumption the optimal active constraints are eventually identified if the projected gradient converges to zero [32]. Calamai and More studied the converging properties of GP and the results were applied for linearly constrained problems [33]. Xu provided an operator oriented mapping approach for providing strong convergence for GPA [34]. Based on the penalty function approach and GPA, Luenberger proposed a new programming algorithm for non-linear constrained optimization [35].

\subsection{Algorithm for Fast Gradient Projection}

The discrete version of the TV (Total Variance) based de-blurring consists of solving an unconstrained convex minimization problem of the form given in Eq. (1).

$$
\min _{x}\|x-a\|_{F}^{2}+2 \lambda|x|_{T V}
$$

where $x$ is the desired unknown image, $a$ is the observed noisy data and $\lambda$ is the regularization parameter with $\lambda>0$.

Let us consider $C=B_{l, u}$, then $P_{c}=P_{B l, u}$. Thus the GP algorithm can be described in the matrix form shown in Eq. (2).

$$
P_{B_{l, u}}(x)_{i, j}= \begin{cases}l & x_{i, j}<l \\ x_{i, j} & l \leq x_{i, j} \leq u \\ u & x_{i, j}>u\end{cases}
$$

where, $l$ is the vector of lower bounds, $u$ is the vector of upper bounds. If $(p, q) \in P$, then the optimal solution of the dual problem with TV based de-noising is described as Eq. (3).

$$
\begin{gathered}
\min _{(p, q) \in P}\left\{h(p, q) \equiv-\left\|H_{c}(a-\lambda \complement(p, q))\right\|_{F}^{2}+\left\|a-\lambda{ }_{\complement}(p, q)\right\|_{F}^{2}\right\} \\
\text { Here, } H_{c}=x-P_{c}(x) \text { for every } x
\end{gathered}
$$

When $T V=T_{1}$, Eq. (3) is given by

$$
x=P_{c}(a-\lambda \complement(p, q))
$$

where $P_{p}$ is the projection operator which maps a matrix pair $(p, q)$ with another matrix pair $(r, s)=P_{p}(p, q)$ and can be readily implemented [36] 
For a better rate of convergence, the Fast Gradient Projection (FGP) method is used on the dual problem stated in Eq. (3). Considering, $\left(r_{1}, s_{1}\right)=\left(p_{0}, q_{0}\right)$ at step 0 , and $k=1,2, \ldots . N$, at step $k, \alpha_{k+1}$ is a sequence of step sizes, Eq. (3) can be denoted as Eqs. (6) and (7).

$$
\begin{gathered}
\left(p_{k+1}, q_{k+1}\right)=P_{p}\left[\left(r_{k+1}, s_{k+1}\right)+\frac{1}{8 \lambda} \complement^{T}\left(P_{c}\left[a-\lambda \complement\left(r_{k+1}, s_{k+1}\right)\right]\right)\right] \\
\alpha_{k+2}=\frac{1+\sqrt{1+4 \alpha_{k+1}^{2}}}{2} \\
\left(r_{k+2}, s_{k+2}\right)=\left(p_{k+1}, q_{k+1}\right)+\left\{\left(\alpha_{k+1}-1\right) /\left(\alpha_{k+2}\right)\right\}\left(p_{k+1}-p_{k}, q_{k+1}-q_{k}\right)
\end{gathered}
$$

\subsection{Method for Centerline Straightening}

Figure 1 shows the schematic representations of the steps involved in Centreline straightening and are summarized below:

Step 1 The locus of the centerline of the vessel is determined by a routine move along the vessel in few pixels at a time. At each point, the intensities of neighboring pixels are measured to determine the local width of the vessel and the position of the next point of the estimated centerline.

Step 2 The locus of the centerline is smoothed using a customized smoothing function.

Step 3 Intensity profiles of the vessel are taken at each point, with the direction of the profile set perpendicular to the local tangent of the locus.

Step $4 \quad$ Each point on the locus is moved by a small displacement to make the profile more symmetrical.

Step 5 When the locus has been optimized, an interpolation between the points generates a new locus in which the points are separated by one pixel. Intensity profiles are calculated as described in Step 3 and used to generate the straightened image. 

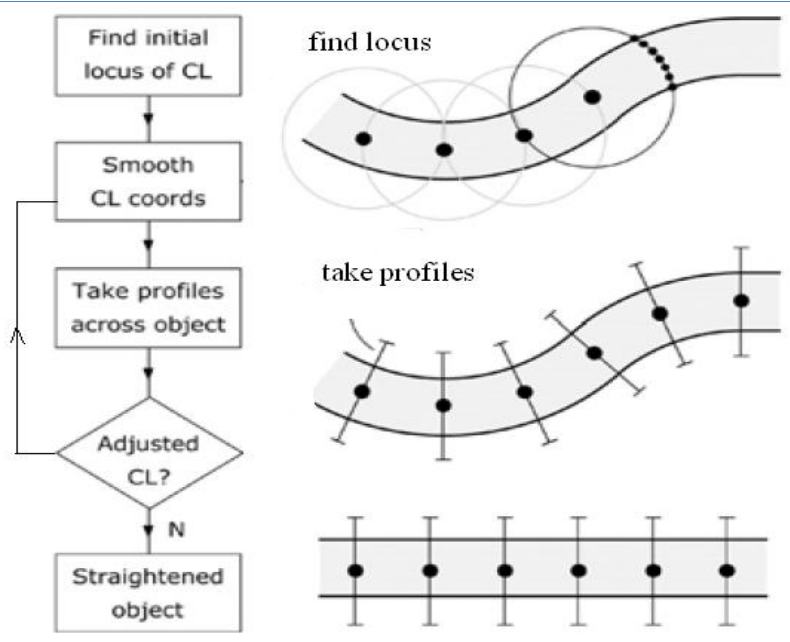

Figure 1. Schematic representation of straighten the blood vessel. The flow diagram on the left side shows how the algorithm loops until a satisfactory result has been achieved (CL refers to the centerline of the vessel).

Steps are illustrated on the right side, where the large points are in (or close to) the centerline of the vessel.

Integration of line primitives is performed in the original vector space. To straighten them, a parameterization of the points is created using the local bases from the moving frame. This algorithm performs a piecewise reformation of a line by using the planes defined by the tangent of the moving frame. These planes are defined in a discrete number of equidistant points along the centerline. To create a straightening of a line, integrated from a seed point lying in the tangent plane, we computed all the intersection points of the line with the planes it intersects during the integration, and then transform these intersections from the vector space into the straightening space. This alignment also allows the combination of visualization of the actual data (such as standard flow visualization techniques) with more abstract visualization techniques like the line graph plot of certain quantities along the centerline.

\section{Tools and Data}

The Straightening algorithms were developed in MATLAB 2011b installed in Pentium-IV $3 \mathrm{GHz}$ processor PC with Window XP operating system. The algorithms developed are tested on various MR images obtained in the DICOM file format. The MR images used in this study were taken from Siemens Esaote ARTOSCAN C MRI Machine with a magnetic field intensity of $0.15 \mathrm{~T}$ and sample per pixel value 1 attained with $63.677701 \mathrm{~Hz}$ imaging frequency.

\section{Results}

The algorithm was tested on more than $100 \mathrm{MR}$ images. Here we showed two Noisy (MRBvessel and BRTBvessel) MR images and Smoothing the image using the FGP filtering method that replaces each pixel with the average of its neighborhood. It reduces the Rician noise and increases the contrast of the MR image. A comparative study was attempted for calculating the performance of the developed filters, with existing filtering techniques such as Median filter (MF), Wiener filter (WF), Hybrid median filter (HMF) using the quality metrics such as Peak Signal to Noise Ratio (PSNR), Mean Structure Similarity Index Matrix (MSSIM) and Contrast to Noise Ratio (CNR). 
Then, the blood vessels are extracted using the Binarization method for easy visual inspection of vessels from the background tissue images. Individual vessels are selected for straightening by assigning the pixel values. Flattening can be considered of as cutting one side of the vessel wall along the main axis and folding it open to reveal the interior. Centerline can be detected using the tangent function and then the standard visualization techniques can be applied for straightening the vessel along the centerline of the object. The smoothed,

The two Noisy (MRBvessel and BRTBvessel) MR images and their corresponding filtered images, binarized and straightened blood vessel MR images are shown in Figs. 2 and 3. The quality metrics, estimated for these images are given in Tables 1 and 2 respectively.

\section{Discussions}

From the visual inspection of the smoothed, binarized and straightened blood vessels in MR images by trained radiologists, the following inferences are arrived at:

1. An FGP filtered image exhibits better smoothing, more homogeneity, and contrast improvements over the other filtering methods quantitaively.

2. Extracted RGB Blood vessels are viewed clearly and it is easy to count the vessels within the MR image.

3. Separately detected the infected blood vessel in Tumor and Cancer cells in extracted RGB blood vessels

4. A cropped grayscale image of the vessel is used to select the particular blood vessel and radiologists can view more conveniently the structure of the vessel and problems developed in this vessel.

5. The straightened blood vessel is used to calculate the length and width of the individual vessel and also to compute the tumor affected vessel area.

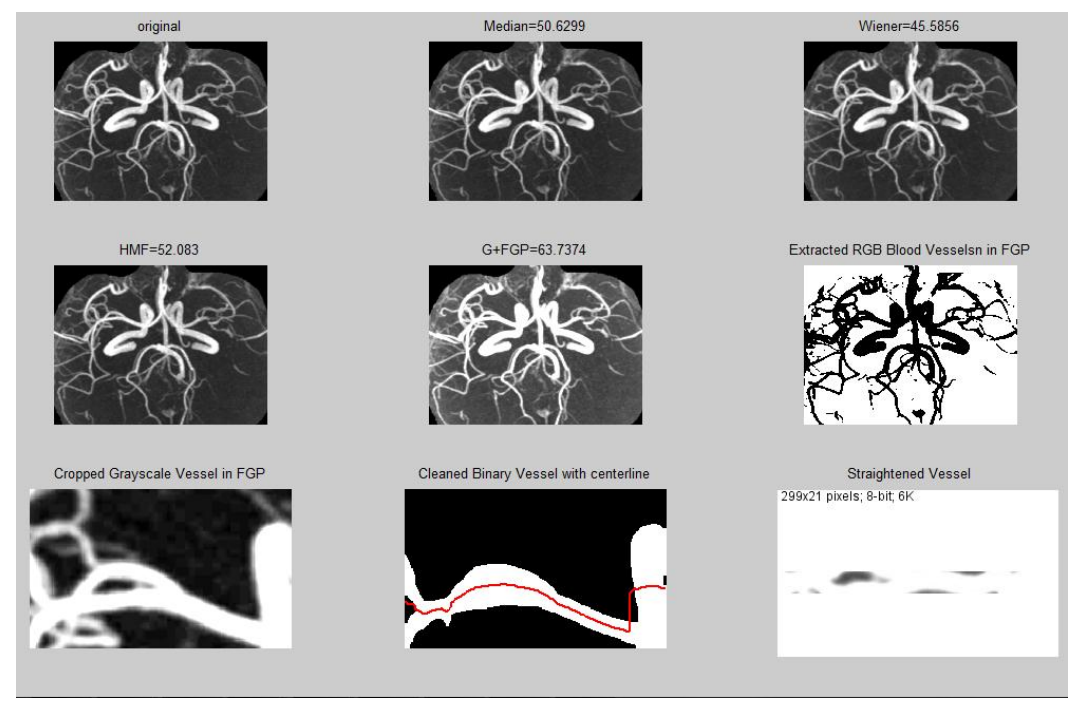

Figure 2. Raw, Smoothed, Extracted and Straightened blood vessels of MRBvessel MR Image. 
Jayabal Papitha and Damodaran Nedumaran; Rician Noise Removal and Straightening of Blood Vessel in MR Images. Journal of Biomedical Engineering and Medical Imaging, Volume 2, No 6, December (2015), pp 28-38



Figure 3. Raw, Smoothed, Extracted and Straightened blood vessels of BRTBvessel MR Image.

Table 1. Estimated Quality Metrics for MR image of MRBvessel

\begin{tabular}{|c|c|c|c|c|}
\hline Filters / Parameters & FGP & Median & Wiener & HMF \\
\hline PSNR & 63.7374 & 50.6299 & 45.5856 & 52.0830 \\
\hline MSSIM & 0.9962 & 0.9935 & 0.9806 & 0.9954 \\
\hline CNR & 1.2293 & $2.6397 \mathrm{e}-006$ & $2.5898 \mathrm{e}-006$ & $4.2911 \mathrm{e}-006$ \\
\hline
\end{tabular}

Table 2. Estimated Quality Metrics for MR image of BRTBvessel

\begin{tabular}{|c|c|c|c|c|}
\hline Filters / Parameters & FGP & Median & Wiener & HMF \\
\hline PSNR & 64.9152 & 34.5163 & 34.0282 & 34.2403 \\
\hline MSSIM & 0.9969 & 0.8343 & 0.8109 & 0.8056 \\
\hline CNR & 1.3533 & $2.5935 \mathrm{e}-004$ & $3.2640 \mathrm{e}-005$ & $4.6144 \mathrm{e}-004$ \\
\hline
\end{tabular}

\section{Conclusion}

In this work, we implemented the Fast Gradient Projection (FGP) Filter Technique for removing the Rician noise present in MRI images and extracted the vessels using binarization techniques. Further, the blood vessels are cropped and straightened using centerline straightening techniques. The algorithm was tested in typical MR images and the results showed that the FGP removes the signal dependent bias effectively and improved the contrast of the image. The performance of the extracted and straightened blood vessels is more convenient to the radiologist for visual analysis. The results of this study reveal that the smoothed, extracted and straightened images are very much useful for the diagnosis of tissue characterization and tumor detection. In the future scope of this study, the aforementioned techniques will be implemented in the DSPs environment for real time applications.

\section{REFERENCES}

[1] Donnell TO, Gupta A, Boult T. A new model for the recovery of cylindrical structures from medical image data. CVRMed-MRCAS 1997;1205:223-32.

[2] Barrett SD, Carvalho CRD. A software tool to straighten curved chromosome images. Chromosome Research Kluwer Academic Publishers 2003;11:83-8. 
[3] Zhang S, Levin DC, Halpern EJ, Fischman D, Savage M, Walinsky P. Accuracy of MDCT in Assessing the Degree of Stenosis Caused by Calcified Coronary Artery Plaques. AJR Am J Roentgenol 2008;191(6):1676-83.

[4] Chuang CL, Chen CM. Accurately and Practical Image Segmentation Algorithm in Coronary Artery. IFMBE Proceedings;14:2483-7.

[5] Kanitsar A, Wegenkittl R, Fleischmann D, Groller ME. Advanced Curved Planar Reformation: Flattening of Vascular Structures. IEEE Transaction on Visualization 2003, VIS 2003;43-50.

[6] Kanitsar A, Fleischmann D, Wegenkittl R, Felkel P. CPR - curved planar reformation. IEEE Transaction on Visualization, USA 2002;37-44.

[7] Wang Y. Blood Vessel Segmentation and shape analysis for quantification of Coronary Artery Stenosis in CT Angiography. School of Engineering \& Mathematical Sciences Dissertations, City University London 2011.

[8] Wang C. Computer-Assisted Coronary CT Angiography Analysis Curved MPR. Linköping University Medical Dissertations, Sweden 2011;1237.

[9] Zhang DP. Coronary Artery Segmentation and Motion Modelling. Department of Computing Dissertations, Imperial College London 2010.

[10] Wink O, Hecht HS, Ruijters D. Coronary Computed Tomographic Angiography in the Cardiac Catheterization Laboratory: Current Applications and Future Developments. Advances in Coronary Angiography, Cardiology Clinics 2009;27(3):513-29.

[11] Saran A. N., Nar F., and Saran M., Vessel segmentation in MRI using a variational image subtraction approach, Turkish Journal of Electrical Engineering \& Comp Sciences, 2014, 22: 499-516.

[12] Lawler LP. CT scanning of the coronary arteries: How to do it and how to interpret it. Appl Radiol 2005;34(10):8-16.

[13] Kanitsar AM. Curved Planar Reformation for Vessel Visualization. Institute for Computer Graphics and Algorithms Dissertation, Vienna University of Technology 2004.

[14] Kim Do-Yeon, 3D volume extraction of cerebrovascular structure on brain magnetic resonance angiography data sets, J. Biomedical Science and Engineering, 2012, 5: 574-9.

[15] Olabarriaga SD, Breeuwer M, Niessen WJ. Evaluation of Hessian-based filters to enhance the axis of coronary arteries in CT images. International Congress Series 2003;1256:1191-6.

[16] Marco Antonio Zenteno M A., Santos-Franco J A., Moscote-Salazar L R., Lee A., Endovascular remodeling of tortuous cervical segments of the internal carotid artery that hinder the 
Jayabal Papitha and Damodaran Nedumaran; Rician Noise Removal and Straightening of Blood Vessel in MR Images. Journal of Biomedical Engineering and Medical Imaging, Volume 2, No 6, December (2015), pp 28-38

management of complex intracranial aneurysms, Romanian Neurosurgery, 2013, XX(3): 24757.

[17] Dupej J., Blood Vessel Visualization on CT Data, WDS'12 Proceedings of Contributed Papers, 2012, Part I, 88-93.

[18] Ropinski T, Hermann S, Reich R, Schafers M, Hinrichs K. Multimodal Vessel Visualization of Mouse Aorta PET/CT Scans. IEEE Trans Vis Comput Graph 2009;15(6):1515-22.

[19] Hénault DR, Sundar H, Cheriet M. Nonrigid 2D/3D Registration of Coronary Artery Models With Live Fluoroscopy for Guidance of Cardiac Interventions. IEEE Transactions on Medical Imaging 2012;31(8):1557-72.

[20] Kocsis E, Trus BL, Steer CJ, Bisher ME, Steven AC. Straighten Curved Objects. J. Struct. Biol 1991;107:6-14.

[21] Wassermann D, Adad JC, Lehericy S, Benali H, Rossignol S, Deriche R, Straightening the Spinal Cord using Fiber Tractography. IEEE International Symposium on Biomedical Imaging: From Nano to Macro 2010;1377-80.

[22] Angelelli P, Hauser H. Straightening Tubular Flow for Side-by-Side Visualization. IEEE Transactions on Visualization and Computer Graphics 2011;17(12):2063-70.

[23] Mistelbauer G, Morar A, Varchola A, Schernthaner R, Baclija I, Kochl A, Kanitsar A, Bruckner S, Groller E. Vessel Visualization using Curvicircular Feature Aggregation Existing. Eurographics Conference on Visualization (EuroVis), Computer Graphics Forum 2013;32(3):231-40.

[24] Lee N, Rasch M. Tangential curved planar reformation for topological and orientation invariant visualization of vascular trees. Conf Proc on IEEE Engg Med Biol Soc 2006;1:1073-76.

[25] Goldstein AA. Convex programming in Hilbert space. Bull. Amer. Math. Soc 1964;70:709-10.

[26] Levitin ES, Polyak BT. Constrained minimization problems. U.S.S.R. Comput. Math. Phys. 1966;6(5):1-50.

[27] Bertsekas DP. Nonlinear programming. Athenas Scientific 1995;22-75 and 223-72.

[28] Bertsekas DP. On the Goldstein - Levitin - Polyak Gradient Projection Method. IEEE Transactions on Automatic Control 1976;21(2):174-84.

[29] Bertsekas DP. Projected Newton methods for optimization problems with simple constraints. SIAM J. Control and Optimization 1982;20(2):221-46.

[30] Gafni EM, Bertsekas DP. Convergence of a Gradient Projection Method. Massachusetts Institute of Technology. Laboratory for Information and Decision Systems 1982;1-12. 
[31] Dunn JC. On the convergence of projected gradient processes to singular critical points. J. Optim. Theory. Appl 1987;55(2):203-16.

[32] Burke JV, More JJ. On the identification of active constraints. SIAM J. Numer. Anal 1988;25(5):1197-211.

[33] Calamai PH, More JJ. Projected Gradient Methods for linearly constrained problems. Mathematical Programming. Springer-Verlag, New York 1987;39(1):93-116.

[34] Xu HK. Averaged Mappings and the Gradient-Projection Algorithm. J. Optim. Theory. Appl. Springer 2011;150:360-78.

[35] Luenberger DG. A Combined penalty function and gradient projection method for nonlinear programming. J. Optim. Theory. App 1974;14(5):477-95.

[36] Soman KP, Ramanathan R. Digital Signal and Image processing- The Sparse Way. Isa Publishers. Elsevier, New Delhi, 2012;408-16. 